\title{
Radio Signals from the DNA: A Philosophical Issue
}

\author{
Bradley Y. Bartholomew \\ Independent Researcher
}

\begin{abstract}
This paper discusses the nature of electromagnetic radio signals and summarizes the advances in optogenetics and radiogenetics that have found a substantial number of genes in both humans and other species that can be activated by electromagnetic signals ranging from extremely low frequency (ELF) radio waves through to infrared radio waves as well as visible light and ultra-violet (UV) tremendously high frequency (THF) radio waves. The paper discusses the advances in nanotechnology where DNA is now being used in its capacity as a nanoscale semiconductor to act as the scaffold for supporting nanoscale integrated circuits. The research findings are presented that the DNA in vivo when it is immersed in water acts as a conducting nanowire with an alternating current (AC). It is proposed that when there is an alternating current (AC) in the DNA it will be emitting electromagnetic radio waves at the same frequency as the alternating current. In addition, it is noted that brainwaves are extremely low frequency (ELF) radio waves and that the DNA as a conducting nanowire would act as a receiver or antenna for these ELF radio waves whereupon an alternating current would be generated in the DNA.
\end{abstract}

Keywords: optogenetics, radiogenetics, Mesmer, brainwaves, EMR, water, ELF, ULF, infrared, ultraviolet

\section{Introduction}

It has been known since the early 1970s that the DNA is emitting electromagnetic radiation (EMR) but what is not appreciated is that this EMR is in the nature of radio signals, and this notwithstanding the fact that the burgeoning new field called optogenetics is uncovering more and more genes that are responsive to EMR and more and more proteins, especially in the neurons of the brain, that are actually synthesized from the DNA and are likewise responsive to EMR. In this paper, I review the pioneering work of Fritz-Albert Popp who was first to popularize the notion that the DNA absorbs and emits light (EMR) and demonstrate how this has mushroomed into the whole new field of study called optogenetics. I then discuss in general terms the basic principles of radio transmission and electronics, and finally I attempt to demonstrate that there is a plethora of evidence in the mainstream literature that the molecular structure and electrical activity in the DNA is such as to make the DNA a classic radio transmitter.

At the outset, I would just like to elucidate a general misconception about radio emissions. It is widely believed that the golden years of radio ended in the 1950s or thereabouts and that radio transmissions only involve the transmission of audio signals, principally voice and music. The fact is that all of modern electronics that involves the transmission of information via EMR are simply different aspects of radio transmissions, including television, cell phones, Wi-Fi, remote control devices, radar, GPS navigation, and wireless Bluetooth devices. If the information is being transmitted by EMR, it's a radio. The radio spectrum extends from Extremely Low Frequency (ELF) waves from 3-30 Hz thru to Tremendously High Frequency (THF) from

Bradley Y. Bartholomew, Independent Researcher, France; main research field: Genetics. 
300-3,000 THz (one THz is one trillion cycles per second) which ends somewhere in the UV range, even higher than visible light. It's an interesting fact that human brain waves are in the same range as the ELF waves, and from the point of view of this paper are considered to be radio waves.

\section{Biophotons}

In 1970, Fritz-Albert Popp was a theoretical biophysicist teaching radiology at the University of Marburg in Germany. Radiology involves the interaction of electromagnetic (EM) radiation on biological systems. At that time, he was working on two almost identical molecules-benzo[a]pyrene and benzo[e]pyrene. This is organic matter, and the former is a lethal carcinogen and the latter is not. There is only a very small difference in their molecular makeup and yet the difference in their toxicity is profound. Popp was observing the different effects of UV light on these molecules.

Popp's experiments were motivated by the findings of a Russian biologist, Alexander Gurwitsch, who in 1923 had proposed that onion roots could communicate with each other using UV light. Gurwitsch had found that onion roots could stimulate the roots of a neighboring plant if they were in a quartz glass pot which allowed UV light to pass through, and not if the pots were made of silicon glass which filtered UV light. As this was the only difference between the two forms of pot, it became apparent to Gurwitsch that the plants must be communicating using ultraviolet light (McTaggart 2008).

So when Popp was using UV light on these benzopyrenes, he found that the toxic version, which is found in coal tar and cigarette smoke among other things, absorbed the light and then re-emitted it at a completely different frequency. The other molecule which is harmless to humans, benzo[e]pyrene allowed the light to pass through unaltered. The carcinogenic molecule appeared to Popp to be a light "scrambler."

So Popp proceeded to perform the same experiment on other compounds, 37 in all, and he got precisely the same result. He found that he was able to predict which substances were carcinogenic from this scrambling effect they had on UV light. In every case they re-emitted the light at a different wavelength. Also, the carcinogenic substances absorbed the light at a specific wavelength- $380 \mathrm{~nm}$ in the ultraviolet range (McTaggart 2008).

Popp went in search of an explanation for this and came across the phenomenon called "photorepair." You can blast a cell with ultraviolet light to the point that it is almost completely destroyed (including the DNA in the nucleus), and then by simply using light of the same frequency but of much weaker intensity the cell can be restored as good as new. In addition to which, Popp knew that this photorepair process is defective in patients with xeroderma pigmentosum. These patients actually die of skin cancer as a result of solar damage because their skin has no ability to repair itself.

It was even known that photorepair works most efficiently at a wavelength of $380 \mathrm{~nm}$, the same frequency that these carcinogens were absorbing light and then scrambling it. Obviously there had to be some connection, or at least it appeared so to Popp, who proceeded to write a groundbreaking paper that was published in a prestigious scientific journal in Germany, where he argued that there must be some kind of light naturally produced by the body that is responsible for photorepair. Furthermore, external substances must cause cancer by absorbing this natural light and scrambling the frequency so it loses its repair capabilities (Popp and Ruth 1979; 1981; Rattemeyer et al. 1981).

Popp came to the conclusion that photons (light) control everything in the cell. He found that all the molecules that make up the cell responded to individual frequencies, and that these molecules in turn 
modulated the frequencies of other processes further down the line. The photons have been likened to the conductor of an orchestra directing all the individual instruments (components in the cell) with his baton. Different frequencies signal all the myriad processes and functions (McTaggart 2008).

These findings by Popp have been the forerunner of a sizeable and ever-growing body of research known as biophotonics or bioinformatics; for instance see the article "The Real Bioinformatics Revolution: Proteins and Nucleic Acids Singing to One Another?" co-authored by Irena Cosic, Professor of Biomedical Engineering, RMIT University, Melbourne, Australia and Dr. Veljko Veljkovic of the Institute of Nuclear Sciences, of Belgrade, Serbia (Cosic and Veljkovic 2007). Hundreds of new chemicals are made by the chemical industry, and these researchers developed a method for predicting whether any of these chemicals may be carcinogenic. Their methods were based on certain electronic properties of the molecules along similar lines to what Popp was doing. It all relates to electromagnetic waves. In addition to determining which chemicals were carcinogenic, they were also able to predict which organic chemical may be mutagenic or toxic, and indeed which organic chemicals may be potentially antibiotic or anticancer agents.

There is so much of conventional genetics and biology that remains unexplained. For instance, how enzymes can recognize their substrates, how antibodies in the immune system can grab onto specific foreign invaders and disarm them, how proteins can "dock" with different partner proteins, or latch onto specific nucleic acids to control gene expression, to mention but a few. The best explanation offered by biologists and geneticists are variations on the so-called "lock and key" model, where molecules randomly bump into each other and, in so doing, they find other molecules with complementary shapes that they can lock into, and thus allow biochemical reactions to take place. The process has been likened generally to finding a friend in a very big crowded ballroom in the dark. In every cell, there can be hundreds of thousands of molecular pair-wise interactions every second, so the conventional explanation of finding the best fit through random collisions is actually no explanation at all (Ho 2008).

Likewise, the explanations of geneticists that segments of DNA are translated and transcribed into proteins are devoid of any specific explanation as to how genetic information actually translates into biological function. The one DNA sequence can encode for several different proteins through multiple splice sites or whatever. Genes and proteins with similar sequences can have totally different functions. Although it is widely accepted that the secondary and tertiary structures of proteins are crucial for their functioning, the base sequences of amino acids that make up the proteins are completely silent as to the how and why of these protein structures. All this information and much more besides must come from some source other than the linear sequence of bases of the DNA molecule. As Dr. Mae-Wan Ho states in her book The Rainbow and the Worm-The Physics of Organisms, "The conventional account is also too mechanical, and at odds with the fuzzy picture of atoms and molecules as 'clouds' of probability density in quantum theory" (Ho 2008).

\section{Optogenetics}

There is a new field of study known as Optogenetics, which started around 2005 with the development of channelrhodopsins, light-activated ion channels that can, with the flick of a switch, instantaneously turn on neurons. These channelrhodopsins were first discovered in unicellular green algae, where they acted as photoreceptors enabling light to guide the movements of these microorganisms. In 2005, these light-sensitive proteins were transplanted into mouse neurons, and the researchers found that by shining a pulse of blue light on these neurons they could reliably trigger action potentials; the ion channel at channelrhodopsin's core opens 
up allowing positively charged ions to enter the cell.

Subsequently another light-sensitive protein, the halorhodopsins, were found to be selective for the negatively charged ion chloride, which would enable neurons to be deactivated by the flick of a switch. Initially this process was found to be very inefficient. Halorhodopsin is what is known as an "ion pump" which means that for every photon of light only one chloride ion crosses the neuron's membrane. Researchers found that it led to only a partial inhibition of neurons in living animals. A breakthrough was announced in April 2014 where researchers were able to develop some genetic mutations of these ion channels, based on their analysis of the molecular structure of the channelrhodopsin, which now gives them a reliable means of silencing the neurons as well (Berndt et al. 2014). According to Science Daily, "The new structurally engineered channel at last gives neuroscientists the tools to both activate and inactivate neurons in deep brain structures using dim pulses of externally projected light" (Science Daily, 2014). Essentially scientists now have the power to activate or deactivate neurons with the flick of a switch.

Since about 2009, the number of research articles appearing using these techniques has been growing exponentially allowing opsin engineering and genomic expansion as well as generalizable opsin targeting strategies, and it is found to have many brain-disease and neuroscience applications, and similar techniques are being used to study stem cells, heart cells, muscle cells and human neurons (Deisseroth 2010).

In 2014, a research paper was published in Nature Genetics which launched a new direction in optogenetics which is known as radiogenetics (Stanley et al. 2014). Radiogenetics will make it possible to remotely control biological targets in living animals without wires, implants, or drugs. A research team at Rockefeller University used electromagnetic waves to turn on insulin production to lower blood sugar in diabetic mice. A naturally occurring iron storage particle in the body, ferritin, when exposed to a radiowave or a magnetic field can activate an ion channel called TRPVI which in turn leads to the activation of an insulin producing gene. These two proteins, ferritin, and TRPVI acting together as a nano-machine can be used to trigger gene expression in vivo. And it is all done by radiowaves.

"The method allows one to wirelessly control the expression of genes in a living animal and could potentially be used for conditions like hemophilia to control the production of a missing protein. Two key attributes are that the system is genetically encoded and can activate cells remotely and quickly," says Jeffrey Friedman, Marilyn M. Simpson Professor head of the Laboratory of Molecular Genetics at Rockefeller University. "We are now exploring whether the method can also be used to control neural activity as a means for noninvasively modulating the activity of neural circuits. (Newswire 2014)

Radiogenetics is being heralded as being more effective than other systems in optogenetics that simply use light as the on/off switch which tend to be only effective near the skin and require permanent implants. Originally the researchers used low-frequency radio waves which heat or move the ferritin particles and the TRPVI which is situated in the membrane surrounding the cell opens up a channel allowing calcium ions to flow through and activate a piece of synthetic DNA implanted upstream to the gene they want to turn on. In this case, it was the gene that synthesizes the protein insulin, but it seems that in this way they can activate any gene of interest (Newswire, 2014). Interestingly the researchers were able to achieve the same result by manipulating the ferritin with a magnetic field, which recalls the theories of Franz Mesmer in the 18th century about animal magnetism and the magnetic cure (Mesmer 1775). It could well be the case that a hypnotist is able to channel his/her brain waves which are ELF radio waves to manipulate the ferritin in the cells and genes of his/her subjects. Mesmer himself stated that the magnets were immaterial to his treatment and what was essential was 
his state of mind towards the patient. He was willing the patient to be healed (Mesmer 2016) and it is well settled that the brainwaves (ELF radiowaves) of healer and patient become synchronized (Fahrion et al. 1992) (Cade and Coxhead 1996).

"The use of a radiofrequency-driven magnetic field is a big advance in remote gene expression because it is non-invasive and easily adaptable," says Johnathan Dordick, who is Howard P. Isermann, professor of Chemical and Biological Engineering and vice president of research at Rensselaer Polytechnic Institute.

You don't have to insert anything — no wires, no light systems — the genes are introduced through gene therapy. You could have a wearable device that provides a magnetic field to certain parts of the body and it might be used therapeutically for many diseases, including neurodegenerative diseases. It's limitless at this point. (Newswire, 2014)

"In this current study, we've shown that by opening the TRPV1 channel to allow calcium ions to enter the cell, we can turn on a gene. Since neurons can be depolarized by calcium and other positively charged ions, such as those the TRPV1 channel controls, we hope that this system may be effective at regulating neural activity," says co-first author Sarah Stanley, a senior research associate in Friedman's lab (Newswire, 2014).

In 1999, Jacqueline Barton and her colleagues at the California Institute of Technology in Pasadena reported that electrons can pass through stretches of DNA by hopping between the overlapping electron clouds of adjacent nucleotide bases. These disc-shaped electron clouds of each individual base form stacks which serve as an electron-rich pathway for conducting electric signals. The distance these signals were said to pass was about 20 nanometers and comprised 60 base pairs of DNA, a stretch long enough to code for 20 amino acids. The researchers reported that DNA promoters, the molecular "switches" that turn on adjacent genes, are typically this length (Nunez et al. 1999). "It's a way of transmitting chemical information over a long distance that's dependent on a DNA sequence," says Barton who suggests that there is an information "superhighway" in the DNA (Coghlan 1999).

This research team also found that there are specific sequences of DNA bases that will act as "insulating" regions and will therefore stop these signals. These regions typically consist of single or multiple pairings between the two DNA bases adenine (A) and thymine (T). "They serve as electron hinges in the circuit," Barton says (Coghlan 1999). Barton speculates that these "electron traps" in the DNA are there to specifically contain the conducting abilities of the DNA within biologically safe limits, and thus prevent damage to the DNA through oxidizing agents (Coghlan 1999). What is clear from this research is that although there is considerable electrical activity in the DNA, the conduction capacities of the DNA are limited. It is suggested that this description of the DNA as facilitating the conduction of electrons along biological significant distances is consistent with the new processes described above in radiogenetics where radio waves are being used to turn on promoter switches that are artificially inserted upstream of the gene of interest. That is to say, the same or similar processes are occurring naturally in the DNA where these promoter switches are turned on by electrical activity.

There is a vast amount of research data about genes being activated by electromagnetic radiation. For instance keying in the search words "genes activated by infrared light" into Google Scholar will produce hundreds of results. Below is an abstract from one paper that was chosen at random and was around number 220 in the list of results (Deguchi et al. 2009). You will get a similar list of results if you search for genes activated by UV light and genes activated by low-frequency radio waves. 
Heat shock promoters are powerful tools for the precise control of exogenous gene induction in living organisms. In addition to the temporal control of gene expression, the analysis of gene function can also require spatial restriction. Recently, we reported a new method for in vivo, single-cell gene induction using an infrared laser-evoked gene operator (IR-LEGO) system in living nematodes (Caenorhabditis elegans). It was demonstrated that infrared (IR) irradiation could induce gene expression in single cells without incurring cellular damage. Here, we report the application of IR-LEGO to the small fish, medaka (Japanese killifish; Oryzias latipes) and zebrafish (Danio rerio), and a higher plant (Arabidopsis thaliana). Using easily observable reporter genes, we successfully induced gene expression in various tissues in these living organisms. IR-LEGO has the potential to be a useful tool in extensive research fields for cell/tissue marking or targeted gene expression in local tissues of small fish and plants.

A research paper published in Nature Communications reports for the first time the use of human brainwaves to control genes, that is to say the brainwaves became radio waves (Fussenegger et al. 2015). The article is entitled "Mind-Controlled Transgene Expression by a Wireless-Powered Optogenetic Designer Cell Implant." The Leader of this research team was a Dr. Martin Fussenegger, a bioengineer at ETH Zurich in Basel, Switzerland. So what did Dr. Fussenegger and his team actually do. He himself states, "We wanted to be able to use brainwaves to control genes. It's the first time anyone has linked synthetic biology and the mind" (Thomson 2014). You will see from the title of this research paper they made use of these techniques being developed in optogenetics of activating genes by EMR, in this case infrared light.

The Fussenegger team implanted some human kidney cells under the skin of a mouse. In these human cells, they had also inserted a gene that was responsive to infrared light. When this gene is activated, it causes a cascade of chemical reactions that will lead to the activation of another gene which we will call their target gene. This is the actual gene they wanted to switch on. Also in the mouse alongside the implanted cells, they put an infrared LED that could be controlled wirelessly.

They then went about getting some characteristic brainwaves from some human volunteers. The volunteers were taught to produce a "relaxed" pattern of brainwaves from meditation techniques, and they played computer games to produce "deep concentration" brainwaves, and they were taught a technique known as "biofeedback" where they learnt to control their thoughts in such a way as to be able to switch on a set of lights on a computer. These human volunteers were wearing EEG devices that were linked wirelessly to the LED implanted in the mouse, and when that was switched on by any one of these three mental states, it activated the light-responsive gene which in turn started the gene cascade and led to the activation of their target gene; hence their claim that human brainwaves can control genes.

The implant in the mouse was encased in a semi-permeable membrane that allowed nutrients from the animal's blood supply to reach the cells inside and also allowed proteins produced in the implant to pass into the bloodstream. When the target gene was activated it produced a human protein that passed into the rodent's bloodstream. Dr. Fussenegger says: "We picked a protein that made an enzyme that was easy to identify in the mouse as proof of concept, but essentially we think we could switch on any target gene we liked" (Thomson 2014).

In this experiment, the Fussenegger team used a standard commercial low-cost Brain-Computer Interface (BCI) headset to record the brain waves of the human subjects. It is stated in his paper that this headset recorded all the normal brain waves, the delta, theta, low alpha, high alpha, beta, and gamma bands. However, in his experiment all this brainwave activity was fed into a meditation meter and it is not specified exactly what 
brainwave frequencies actually resulted in the expression of specific genes. These brain waves were literally extremely low frequency (ELF) radio waves and these were then converted into tremendously high frequency (THF) infrared radio waves which were transmitted wirelessly to the LED implanted in the mouse. Essentially then the whole process involved the transmission of information via radio waves.

\section{DNA as a Radio Transmitter}

In order to understand the DNA as a radio transmitter, you first have to know the difference between direct current (DC) and alternating current (AC). Electric current that flows continuously in a single direction is called a direct current, or DC. That is to say in a direct current circuit, current is caused by electrons that all line up and move in one direction. Within a wire carrying direct current, electrons jump from atom to atom while moving in a single direction. Thus in principle, a given electron that starts out at one end of the wire will eventually end up at the other end of the wire. The explanation for this is voltage or potential difference at the two ends of the wire. The high voltage end has a deficiency of electrons and is said to be positively charged and the low voltage end has a surplus of electrons and is said to be negatively charged. In a wire that is a good conductor, like copper or silver, there is an abundance of electrons that are only loosely attached to their atoms, and thus when a voltage is applied to the wire then these electrons are free to move towards the positively charged end.

In an alternating current on the other hand, the electrons don't move in only one direction. Instead, the current goes in one direction for a period of time, and then the current reverses and goes in the opposite direction for an equal period of time. In other words, the current is said to oscillate backwards and forwards instead of going steadily forwards. An alternating current is achieved when the voltage of the circuit reverses itself. The terminals of the circuit oscillate between high voltage and low voltage and the number of times this oscillation occurs every second is known as the frequency of the circuit and is measured in Hertz (Hz). Alternating current is used in public power distribution networks (including household supply) and in the United States (for instance) the voltage reverses 60 times per second, that is to say the frequency is $60 \mathrm{~Hz}$.

There are natural sources of radio waves, like lightning and astronomical objects, but as a general rule radio waves of whatever frequency are transmitted by alternating current flowing through a conductor, generally known as a transmitter, which gives off electromagnetic waves (EMR) which propagate in all directions at the speed of light, and which have the frequency of the alternating current that generated them. Likewise an antenna or receiver is simply a conductor that picks up these radio waves and an alternating current is generated in the antenna at that same frequency. When radio was invented audio data were inserted into the carrier wave by modulating the amplitude of the oscillations in the carrier wave but the frequency of the carrier wave remained the same. This was known as AM radio. Then FM radio was introduced where the audio data would make slight variations to the frequency of the carrier wave but the amplitude of the carrier wave would remain the same. When television was introduced the carrier wave was used to transmit audio and video data, and since then the list of other types of information being transmitted by radio waves has continued to grow.

Before getting on to the electronic properties of DNA we must first look briefly at infrared radio waves. Infrared light is invisible light between $1 \mathrm{Thz}$ and $400 \mathrm{Thz}$ (one Thz is one trillion cycles per second). In the electromagnetic spectrum, it has a shorter wavelength than microwaves and a longer wavelength than visible light. More than half of the light energy that comes from the sun is infrared light and although it is invisible, it does heat surfaces that are exposed to it. All matter emits some infrared light and that is why infrared detectors 
can be used for night-vision goggles and cameras. Infrared is widely used for wireless communication across small distances, for instance the remote control for televisions and garage doors etc. Infrared radio waves are high frequency radio waves. When you point the remote control at the television and push a button, the remote control turns on the infrared light source and encodes a message on it. The receiver on the television picks up this signal, decodes the message, and does whatever the message directs it to do-turns up the volume, changes the channel, and so on.

The molecular composition of DNA would suggest that it would be a semiconductor. The core DNA base pairs are made up of carbon atoms interspersed with nitrogen, oxygen, and hydrogen atoms. Carbon, like silicon, with four electrons in its valence shell is a classical semiconductor, and the nitrogen and hydrogen atoms would act as doping agents. To "dope" a semiconductor makes it conduct electricity more readily. In fact, the doping of carbon nanotubes and composite graphene structures with nitrogen is very common and comparisons have already been drawn with the DNA (Won et al. 2014). The hydrogen atoms play an important role in the doping process because the hydrogen atom readily donates its sole electron to the nitrogen atoms which means that throughout the DNA molecule the nitrogen atoms are slightly negatively charged and the hydrogen atoms are slightly positively charged (Guerra et al. 1999). This means that the base-pair components of the DNA double helix themselves present as both a $\mathrm{p}$ and $\mathrm{n}$ semiconductor, that is to say it has both electrons and "holes" that are readily available for conduction. In addition, the DNA double helix has a sugar-phosphate backbone and since the phosphate groups in the backbone are negatively charged, the DNA is usually surrounded by positive "counterions." The DNA is said to be "ideal for electron transfer" (Dekker and Ratner 2001).

Initially, there was a great deal of controversy whether the DNA molecule was actually a metal or a conducting nanowire or even a superconductor (Dekker and Ratner 2001). Basically, a metal has electrons always available in the conduction band and all you have to do is applying a voltage and current will flow. With a semiconductor, there is a large gap between the energy levels of the valence band and the conduction band and energy has to be applied to the material to make the electrons jump into the conduction band, and only then will a current flow if a voltage is applied. A conducting nanowire is a conductor that is only a few nanometers in diameter. A superconductor is a metal with zero resistance which will allow current to flow indefinitely without losing any energy. In fact, levels of resistance have been measured in the DNA which vary widely from one MegaOhm $(1 \mathrm{M} \Omega)$ which indicates that it conducts well right up to 10 trillion ohms $\left(10^{13} \Omega\right)$ for DNA molecules 40 nanometers or longer which means that it is an insulator with infinite resistance (Dekker and Ratner 2001). Overall, it seems certain that resistance in the DNA increases exponentially with distance and that long DNA molecules are true insulators (Dekker and Ratner 2001).

The fact is that the semiconducting properties of DNA as well as other characteristics make it ideal for building nanometer scale integrated circuits. The DNA molecule is only 2 nanometers in diameter and it can be folded into any desired shape. The folded DNA acts as a scaffold to support various nanometer scale components such as electrically-conductive carbon nanotubes. The process is called DNA origami because it is reminiscent of the ancient Japanese art of paper folding. "Think of it a bit like the pegboards people use to organize tools in their garages, only in this case, the pegboard assembles itself from DNA strands and the tools likewise find their own positions," says Paul Rothemund, research professor of bioengineering, computing and mathematical sciences, and computation and neural systems at Caltech. "It all happens in a test tube without human intervention, which is important because all of the parts are too small to manipulate efficiently, and we want to make billions of devices" (Science Daily, 2016a). 
"We would like to use DNA's very small size, base-pairing capabilities and ability to self-assemble, and direct it to make nanoscale structures that could be used for electronics," says Adam T. Woolley, Ph.D. at Brigham Young University. He explains that the smallest features on chips currently produced by electronics manufacturers are 14 nanometers wide. That's more than 10 times larger than the diameter of single-stranded DNA, meaning that this genetic material could form the basis for smaller-scale chips. "The problem, however, is that DNA does not conduct electricity very well," he says. "So we use the DNA as a scaffold and then assemble other materials on the DNA to form electronics" (Science Daily, 2016b).

It turns out that the DNA does conduct electricity over longer distances but only if the molecule is immersed in water (Armitage et al. 2003) or in a phosphate buffer solution (Slinker et al. 2011). This is actually its natural state in vivo where in fact the nitrogen elements are hydrophobic and the sugar-phosphate backbone is hydrophilic. The hydrophobic negatively charged nitrogen atoms are actually what cause the DNA to twist into its characteristic helix shape to prevent the water from getting into the middle of the molecule (Zhao 2011), while the negatively charged sugar-phosphate backbone is actually soluble in water (Westhof 1993). In fact, it has been found that the DNA can only act as a conducting nanowire if it retains its helical structure (Slinker et al. 2011; Brown 2011). When the DNA is in aqueous solution the negatively charged sugar phosphate backbone becomes an electron rich liquid medium which is an excellent conductor of electricity (Turro et al. 1991; Biever 2003). This explains the reports that the DNA acts as a metal for conducting electricity (Dekker and Ratner 2001). The nitrogenous base-pairs being hydrophobic would continue to act as a semiconductor separately from the metal-like conduction of electricity in the surrounding water. We have here a full explanation for the reports that the DNA is a conducting nanowire, as well as the fact that it is conducting an alternating current (AC). "This is exactly what you would expect if water is responsible for the conductivity. Water is a polar molecule, across which electrons can shift to produce an alternating current. But they cannot travel freely from molecule to molecule to produce direct current," says co-author of the report Peter Armitage, Postdoctoral Fellow at UCLA (Biever 2003).

A nanowire conducting alternating current (AC) is going to give off radio waves at the same frequency as the electric current. The DNA in vivo is a transmitter of radio waves and what is more it will act as a receiver or antenna, where it will pick-up radio waves of whatever frequency whereupon an alternating current of the same frequency will be generated in the nanowire.

In 2010, a group of researchers which included Nobel Laureate Dr. Luc Montagnier wrote a paper entitled "DNA Waves and Water" which reports that the DNA changes the molecular structure of the water. Essentially the research found that DNA emits an ultra-low frequency (ULF) electromagnetic signal that can be stored and transmitted in pure water, and then if you introduce the ingredients necessary for synthesizing the DNA by the polymerase chain reaction - nucleotides, primers, polymerase - to the tube with the pure water that had gained the EM signal then the original DNA sequence would be recreated. Most of the discussion in the paper centers around how the nanostructures in the water can retain the information from the DNA in the form of memory but the essential point is the fact that the information about the original sequence of the DNA was emitted by the DNA as an electromagnetic field. These are ULF radio waves that contain information. The fact that it is precisely pure water that has this capacity to transmit the signal from the DNA and to retain the integrity of the signal in memory will not come as a surprise when it is remembered that about $65 \%$ of the composition of our bodies is water. In other words, it is reasonable to assume that this is a normal metabolic process that is occurring naturally in all creatures containing DNA, including plants. In these experiments, a copper solenoid is 
placed around the tubes and they were exposed to a low intensity electric current oscillating at $7 \mathrm{~Hz}$ produced by an external generator. The magnetic field produced by the external generator is maintained for 18 hours at room temperature. This ambient externally applied magnetic field was found to be essential for the EMS transmission in the water tube. The paper concludes with the observation: "DNA signaling is stimulated by 7 $\mathrm{Hz}$ naturally occurring waves on earth. Waves produced by the human brain are also in the range of $7 \mathrm{~Hz}$. These are interesting questions to be asked and possibly answered" (Montagnier et al. 2010).

This effect was found when the ambient magnetic field was at a minimum of $7 \mathrm{~Hz}$. In other words, the magnetic field oscillating at less than $7 \mathrm{~Hz}$ did not cause the effect. It is not stated what magnetic fields oscillating higher than $7 \mathrm{~Hz}$ were tested. The naturally occurring waves on Earth, the so-called Schumann resonance has a fundamental frequency of $7.83 \mathrm{~Hz}$ (Rusov et al. 2012) and the alpha brain waves and the mu rhythm have a bandwidth from 7.5 to $12.5 \mathrm{~Hz}$ (Gerrard and Malcolm 2007). The alpha brain waves occur when we are in an aware and relaxed state, and the mu rhythm extends across the motor cortex at the back of the head from ear to ear and is responsible for our motor actions. These are all classic ULF radio waves operating in the same frequency window. For instance, this ambient magnetic field in the research paper oscillating at $7 \mathrm{~Hz}$ is emitting ULF radio waves at that frequency and the DNA sample acting as an antenna would pick up those radio waves which would generate an alternating current $\mathrm{AC}$ in the DNA at that precise frequency. This is what has "stimulated the DNA signaling." Incidentally, the alpha brain waves and the mu rhythm are widely used in cybernetics, to make man-machine interfaces and Brain-Computer Interfaces (BCIs) precisely because they are ULF radio waves that are transmitting information (Inoue et al. 2008; Hundia 2015).

\section{Conclusion}

In this paper, I have attempted to demonstrate that the DNA is both emitting and receiving radio waves. This was originally the discovery of Fritz-Albert Popp in the 1970s who reported a frequency range in the vicinity of visible light and UV light. Since then the fields of optogenetics and radiogenetics have found that the DNA is also responsive to THF infrared radio waves as well as ULF ultra-low frequency radio waves. In addition, it is now known that the DNA is being used as a scaffold for building integrated circuits on the nanoscale and that the DNA in vivo immersed in water acts as a nanowire conducting alternating current (AC). This being the case it is inevitable that the DNA is giving off electromagnetic radio waves at the same frequency as the alternating current it is conducting. It has also been demonstrated in this paper that the brain waves are also in the range of ELF radio waves as well as the Schumann resonance which means that all these electromagnetic waves would be capable of generating an alternating current in the DNA by virtue of the fact that it is a conducting nanowire.

\section{Works Cited}

Armitage, N. P., Briman, M., and Gruner, G. "Charge Transfer and Charge Transport on the Double Helix." Physica Status Solidi (b) 241 (2003): 69.

Berndt, A., Soo, Y. L., Ramakrishnan, C., and Deisseroth, K. "Structure-Guided Transformation of Channelrhodopsin into a Light-Activated Chloride Channel." Science, 25 April 2014.

Biever, C. "DNA — Celebrating 50 Years of the Double Helix." New Scientist, March 15, 2003.

Brown, M. "Electrons Charge down DNA Molecular Wire." Chemistry World, Royal Society of Chemistry 30 January 2011. $<$ http://www.rsc.org/chemistryworld/News/2011/January/30011102.asp>. 
Cade, M. and Coxhead, N. The Awakened Mind: Biofeedback and the Development of Higher States of Awareness. London: Element Books reprint, 1996.

Coghlan, A. "Electric DNA.” New Scientist, 13 February, 1999.

Cosic, I. and Veljkovic, V. "The Real Bioinformatics Revolution: Proteins and Nucleic Acids Singing to One Another?" Press Release of Institute of Science in Society. 02/02/2007. <http://www.i-sis.org.uk/TheRealBioinformaticsRevolution.php>.

Dekker, C. and Ratner M. A. "Electronic Properties of DNA." Physics World, August 2001.

Deguchi, T., Itoh, M., Urawa, H., Matsumoto, T., Nakayama, S., Kawasaki, T., Kitano, T., Oda, S., and Mitani, H. "Infrared Laser-Mediated Local Gene Induction in Medaka, Zebrafish and Arabidopsis Thaliana." Development Growth \& Differentiation 51.9 (2009).

Deisseroth, K. “Optogenetics.” Nature Methods 8.1 (2011).

Fahrion, S., Wirkus, M., and Pooley, P. "EEG Amplitude, Brain Mapping, \& Synchrony in \& between a Bioenergy Practitioner \& Client during Healing." Subtle Energies \& Energy Medicine $3.1 \quad$ (1992). $<$ http://journals.sfu.ca/seemj/index.php/seemj/article/view/144>.

Fussenegger, M., Folcher, M., Oesterle, S., Zwicky, K., Thekkottil, T., Heymoz, J., Hohmann, M., Christen, M., Daoud El-Baba, M., and Peter Buchmann, P. "Mind-Controlled Transgene Expression by a Wireless-Powered Optogenetic Designer Cell Implant." Nature Communications 5 (2015): 5392.

Gerrard, P. and Malcolm, R. "Mechanisms of Modafinil: A Review of Current Research." Journal of Neuropsychiatric Disease and Treatment 3.3 (2007): 349-64.

Guerra, C. F., Bickelhaupt, M., Snijders, J. G., and Baerends, E. J. "The Nature of the Hydrogen Bond in DNA Base Pairs: The Role of Charge Transfer and Resonance Assistance.” Chemistry—A European Journal 5.12 (1999): 3581-94.

Ho, M-W. The Rainbow and the Worm-The Physics of Organisms. Singapore: World Scientific Publishing Co., 2008.

Hundia, R. "Brain Computer Interface-Controlling Devices Utilizing the Alpha Brain Waves." International Journal of Scientific \& Technology Research 4.1 (2015).

Inoue, S., Akiyama, Y., Izumi, Y., and Nishuima, S. "The Development of BCI Using Alpha Waves for Controlling the Robot Arm.” IEICE Transactions on Communications Vol. E91-B No.7 (2008): 2125-32.

McTaggart, L. The Field: The Quest for the Secret Force of the Universe. New York: Harper Collins, 2008.

Mesmer, F. Sendschreiben an einen auswärtigen Arzt über die Magnetkur (Circulatory letter to a foreign physician about the magnetic cure/original language: German). Didot, Genf und Paris, 1775.

Mesmer, F. Mesmerism: The Discovery of Animal Magnetism. Seattle: Create Space Independent Publishing Platform, 2016.

Montagnier, L., Aissa, J., Del Giudice, E., Lavallee, C., Tedeschi, A., and Vitiello, G. "DNA Waves and Water." Journal of Other Quantitative Biology 1012.5166v1 (2010).

Núñez, M. E., Hall, D. B., and Barton, J. K. "Long-Range Oxidative Damage to DNA: Effects of Distance and Sequence." Chemistry \& Biology 6.2 (1999): 85-97.

News Wire. 2014. < http://newswire.rockefeller.edu/2014/12/15/radiogenetics-seeks-to-remotely-control-cells-and-genes/>.

Popp, F-A. and Ruth, B., eds. Electromagnetic Bioinformation. Baltimore: Urban \& Schwarzenburg, 1979.

---. "Emission of Visible and Ultraviolet Radiation by Active Biological Systems." Collective Phenomena 3 (1981): 187-214.

Rattemeyer, M., Popp, F. A., and Nagl, W. "Evidence of Photon Emission from DNA in Living Systems.” Naturwissen 68 (1981): 572-80.

Rusov, V. D., Lukin, K. A., Zelentsova, T. N., Linnik, E. P., Beglaryan, M. E., Smolyar, V. P., Filippov, M., and Vachev, B. “Can Resonant Oscillations of the Earth Ionosphere Influence the Human Brain Biorhythm?” 2012. arXiv:1208.4970 [physics.gen-ph]. <https://arxiv.org/abs/1208.4970>.

Slinker, D. J., Muren, N. B., Renfrew, S. E., and Barton, J. K. "DNA Charge Transport over 34 nm.” Nature Chemistry 3 (2011): 228-33.

Stanley, S. A., Sauer, J., Kane, R. S., Dordick, J. S., and Friedman, J. M. "Remote Regulation of Glucose Homeostasis Using Genetically Encoded Nanoparticles.” Nature Medicine 21 (2014): 92-98.

Science Daily. 2014. <https://www.sciencedaily.com/releases/2014/04/140424140905.htm>.

---. 2016a. <https://www.sciencedaily.com/releases/2016/07/160712133919.htm>.

---. 2016b. <https://www.sciencedaily.com/releases/2016/03/160314084824.htm>.

Thomson, H. "Human Thoughts Used to Switch on Genes." New Scientist, 15 November, 2014.

Turro, N. J., Barton, J. K., and Tomalia, T. A. "Molecular Recognition and Chemistry in Restricted Reaction Spaces. Photophysics and Photoinduced Electron Transfer on the Surfaces of Micelles, Dendrimers, and DNA." Accounts of Chemical Research 24.11 (1991): 332-40. 
Won, J. L., Uday, N. M., Ju, M. L., Joonwon, L., Tae, H. H., and Sang, O. K. "Nitrogen-Doped Carbon Nanotubes and Graphene Composite Structures for Energy and Catalytic Applications." Chemical Communications 50 (2014): 6818-30.

Westhof, E. "Structural Water Bridges in Nucleic Acids." Water and Biological Macromolecules. London: Macmillan Education UK, 1993. 226-43.

Zhao, X. "Self-assembly of DNA Segments on Graphene and Carbon Nanotube Arrays in Aqueous Solution: A Molecular Simulation Study." Journal of Physical Chemistry C 115.14 (2011): 6181-9. 